\title{
A relation between the Laplacian and signless Laplacian eigenvalues of a graph
}

\author{
Saieed Akbari • Ebrahim Ghorbani • \\ Jack H. Koolen • Mohammad Reza Oboudi
}

Received: 24 November 2009 / Accepted: 23 March 2010 / Published online: 7 April 2010

(C) Springer Science+Business Media, LLC 2010

\begin{abstract}
Let $G$ be a graph of order $n$ such that $\sum_{i=0}^{n}(-1)^{i} a_{i} \lambda^{n-i}$ and $\sum_{i=0}^{n}(-1)^{i} b_{i} \lambda^{n-i}$ are the characteristic polynomials of the signless Laplacian and the Laplacian matrices of $G$, respectively. We show that $a_{i} \geq b_{i}$ for $i=0,1, \ldots, n$. As a consequence, we prove that for any $\alpha, 0<\alpha \leq 1$, if $q_{1}, \ldots, q_{n}$ and $\mu_{1}, \ldots, \mu_{n}$ are the signless Laplacian and the Laplacian eigenvalues of $G$, respectively, then $q_{1}^{\alpha}+\cdots+q_{n}^{\alpha} \geq \mu_{1}^{\alpha}+\cdots+\mu_{n}^{\alpha}$.
\end{abstract}

Keywords Laplacian · Signless Laplacian · Incidence energy · Laplacian-like energy

S. Akbari - E. Ghorbani · M.R. Oboudi

Department of Mathematical Sciences, Sharif University of Technology, P.O. Box 11155-9415,

Tehran, Iran

S. Akbari

e-mail: s_akbari@sharif.edu

E. Ghorbani

e-mail: e_ghorbani@math.sharif.edu

M.R. Oboudi

e-mail: m_r_oboudi@math.sharif.edu

S. Akbari · E. Ghorbani - M.R. Oboudi

School of Mathematics, Institute for Research in Fundamental Sciences (IPM),

P.O. Box 19395-5746, Tehran, Iran

J.H. Koolen $(\bowtie)$

Department of Mathematics, POSTECH, Pohang 790-785, South Korea

e-mail: koolen@postech.ac.kr

J.H. Koolen

Pohang Mathematics Institute (PMI), POSTECH, Pohang 790-785, South Korea 


\section{Introduction}

Let $G$ be a graph with vertex set $V(G)=\left\{v_{1}, \ldots, v_{n}\right\}$ and edge set $E(G)=$ $\left\{e_{1}, \ldots, e_{m}\right\}$. The adjacency matrix of $G, A=\left(a_{i j}\right)$, is an $n \times n$ matrix such that $a_{i j}=1$ if $v_{i}$ and $v_{j}$ are adjacent, and otherwise $a_{i j}=0$. The incidence matrix of $G$, denoted by $X=\left(x_{i j}\right)$, is an $n \times m$ matrix whose rows are indexed by the set of vertices of $G$ and columns are indexed by the set of edges of $G$ defined by

$$
x_{i j}:= \begin{cases}1 & \text { if } e_{j} \text { is incident with } v_{i} \\ 0 & \text { otherwise }\end{cases}
$$

If we consider an orientation for $G$, then in a similar manner as the incidence matrix, the directed incidence matrix of the (oriented) graph $G$, denoted by $D=\left(d_{i j}\right)$, is defined as

$$
d_{i j}:= \begin{cases}+1 & \text { if } e_{j} \text { is an incoming edge to } v_{i} \\ -1 & \text { if } e_{j} \text { is an outgoing edge from } v_{i} \\ 0 & \text { otherwise }\end{cases}
$$

Let $\Delta$ be the diagonal matrix whose entries are the degrees of vertices of $G$. It is well known that $D D^{\top}=\Delta-A$ is the Laplacian matrix of $G$ denoted by $L$ and $X X^{\top}=\Delta+A$ is the signless Laplacian $G$ denoted by $Q$. Since $L$ and $Q$ are symmetric matrices, their eigenvalues are real. We denote the eigenvalues of $L$ and $Q$ by $\mu_{1}(G) \geq \cdots \geq \mu_{n}(G)$ and $q_{1}(G) \geq \cdots \geq q_{n}(G)$, respectively (we drop $G$ when it is clear from the context). The matrices $L$ and $Q$ are similar if and only if $G$ is bipartite (see [4]). The incidence energy $\operatorname{IE}(G)$ of the graph $G$ is defined as the sum of singular values of the incidence matrix [8]. The Laplacian-energy like invariant $\operatorname{LEL}(G)$ is defined as the sum of square roots of the Laplacian eigenvalues [9]. In other words,

$$
\operatorname{IE}(G)=\sum_{i=1}^{n} \sqrt{q_{i}(G)}, \quad \text { and } \quad \operatorname{LEL}(G)=\sum_{i=1}^{n} \sqrt{\mu_{i}(G)}
$$

The connection between IE and Laplacian eigenvalues was first pointed out in [5]. For more information on IE and LEL, see [6] and the references therein.

Our motivation of this work is the following conjecture.

Conjecture 1 ([1]) For any graph $G$ of order n, we have the following:

$$
\sqrt{q_{1}}+\cdots+\sqrt{q_{n}} \geq \sqrt{\mu_{1}}+\cdots+\sqrt{\mu_{n}}
$$

The conjecture is equivalent to say that, for any graph $G$,

$$
\operatorname{IE}(G) \geq \operatorname{LEL}(G) .
$$

In this paper we confirm this conjecture by proving the more general statement that for any $0<\alpha \leq 1$,

$$
q_{1}^{\alpha}+\cdots+q_{n}^{\alpha} \geq \mu_{1}^{\alpha}+\cdots+\mu_{n}^{\alpha}
$$


To settle this result, we make use of a property of elementary symmetric functions as described below. We remark that the quantity $\mu_{1}^{\alpha}+\cdots+\mu_{n}^{\alpha}$ has been studied recently (see $[10,11])$ and is denoted by $S_{\alpha}(G)$.

The elementary symmetric functions of real numbers $x_{1}, \ldots, x_{n}$ are the values

$$
c_{k}\left(x_{1}, \ldots, x_{n}\right)=\sum_{\substack{S \subseteq\{1, \ldots, n\} \\|S|=k}} \prod_{i \in S} x_{i},
$$

for $k=1, \ldots, n$. We define the ordering $\left(x_{1}, \ldots, x_{n}\right) \preceq\left(y_{1}, \ldots, y_{n}\right)$ if

$$
c_{k}\left(x_{1}, \ldots, x_{n}\right) \leq c_{k}\left(y_{1}, \ldots, y_{n}\right), \quad \text { for all } k=1, \ldots, n .
$$

Efroymson, Swartz, and Wendroff [3] gave a necessary and sufficient condition for a real continuously differentiable function $f$ to satisfy the following property:

$$
\left(x_{1}, \ldots, x_{n}\right) \preceq\left(y_{1}, \ldots, y_{n}\right) \Longrightarrow\left(f\left(x_{1}\right), \ldots, f\left(x_{n}\right)\right) \preceq\left(f\left(y_{1}\right), \ldots, f\left(y_{n}\right)\right) .
$$

They showed that for any real $\alpha$ with $0<\alpha \leq 1$, the function $f(x)=x^{\alpha}$ satisfies (2). In particular:

Theorem 2 ([3]) If $\left(x_{1}, \ldots, x_{n}\right) \preceq\left(y_{1}, \ldots, y_{n}\right)$, then, for any $0<\alpha \leq 1$,

$$
x_{1}^{\alpha}+\cdots+x_{n}^{\alpha} \leq y_{1}^{\alpha}+\cdots+y_{n}^{\alpha} .
$$

Theorem 2 suggests that in order to establish Inequality (1), it is sufficient to prove that

$$
\left(\mu_{1}, \ldots, \mu_{n}\right) \preceq\left(q_{1}, \ldots, q_{n}\right) .
$$

We will do this in Sect. 2. In Sect. 3, two bounds are obtained for the sum of powers of the Laplacian eigenvalues of graphs.

\section{The coefficients of the Laplacian and signless Laplacian polynomials}

Let $M$ be a matrix and $R$ and $S$ be two subsets of the sets of rows and columns of $M$, respectively. By $M(R, S)$ we mean the submatrix of $M$ defined by the rows corresponding to the rows in $R$ and the columns corresponding to the columns in $S$.

Let $G$ be a graph and $S \subseteq E(G)$. By $\langle S\rangle$ we denote the spanning subgraph of $G$ whose edge set is $S$.

Lemma 3 ([2, Lemma 7.4]) Let $G$ be a graph and $R$ and $S$ be non-empty subsets of the vertex set and edge set of $G$ with $|R|=|S|$. Let $V_{0}$ denote the set of vertices of the induced subgraph $\langle S\rangle$. Then $D(R, S)$ is invertible if and only if the following conditions are satisfied:

(i) $R$ is a subset of $V_{0}$.

(ii) $\langle S\rangle$ is a forest.

(iii) $V_{0} \backslash R$ contains precisely one vertex from each connected component of $\langle S\rangle$. 
Lemma 4 Let $G$ be a connected graph of order $n$ with the incidence matrix $X$. Then any set of $n-1$ rows of $X$ is linearly independent.

Proof Let $\left\{v_{1}, \ldots, v_{n}\right\}$ be the vertex set of $G$ and $\mathbf{x}_{1}, \ldots, \mathbf{x}_{n}$ be the rows of $X$, where $\mathbf{x}_{i}$ corresponds to $v_{i}$, for $i=1, \ldots, n$. The case $n=1$ is trivial. Let $n \geq 2$. If $\operatorname{rank} X=n$, then we are done. So, suppose that rank $X \leq n-1$. Let $\left(w_{1}, \ldots, w_{n}\right)$ be a non-zero left null-vector of $X$, that is $w_{1} \mathbf{x}_{1}+\cdots+w_{n} \mathbf{x}_{n}=\mathbf{0}$. Suppose that $w_{j} \neq 0$. If $v_{r}$, for some $r$, is adjacent to $v_{j}$, then $w_{r}=-w_{j}$ because in each column of $X$ there are exactly two non-zero entries. Since $G$ is connected, it follows that for every $i, i=1, \ldots, n, w_{i}= \pm w_{j}$. This means that any non-zero left null-vector of $X$ has no zero entry. Thus, no set of $n-1$ rows of $X$ can be linearly dependent.

Theorem 5 Let $G$ be a graph of order $n$. Let $\sum_{i=0}^{n}(-1)^{i} a_{i} \lambda^{n-i}$ and $\sum_{i=0}^{n}(-1)^{i} b_{i} \lambda^{n-i}$ be the characteristic polynomials of the signless Laplacian and the Laplacian matrices of $G$, respectively. Then

$$
a_{i} \geq b_{i}, \quad \text { for } i=0,1, \ldots, n \text {. }
$$

Equalities hold in (3) for all $i=0,1, \ldots, n$ if and only if $G$ is bipartite.

Proof It is well known that for every $i, 1 \leq i \leq n, b_{i}=\sum_{R} \operatorname{det} L(R, R)$, where the summation is taken over all set of vertices $R$ of cardinality $i$. By the Binet-Cauchy Theorem (see [7, p. 22]),

$$
b_{i}=\sum_{R, S} \operatorname{det} D(R, S)^{2},
$$

where the summation is taken over all subsets $R$ of the vertices and all subsets $S$ of the edges of $G$ with $|R|=|S|=i$. Similarly, one has

$$
a_{i}=\sum_{R, S} \operatorname{det} X(R, S)^{2},
$$

with the summation taken over the same set.

We claim that $\operatorname{det} X(R, S)^{2} \geq \operatorname{det} D(R, S)^{2}$, for every subset $R$ of the vertices and every subset $S$ of the edges of $G$ with $|R|=|S|$. By a result due to Poincaré (see [2, Proposition 5.3]), det $D(R, S) \in\{-1,0,1\}$. Thus it suffices to prove that $X(R, S)$ is invertible whenever $D(R, S)$ is so. Suppose that $D(R, S)$ is invertible. Let $T_{1}, \ldots, T_{k}$ be all connected components of $\langle S\rangle$. By Lemma 3(i), $R \subseteq V_{0}$ where $V_{0}$ is the set of vertices of the subgraph $\langle S\rangle$. Also, by Lemma 3(iii), for $i=1, \ldots, k, V\left(T_{i}\right) \backslash R$ has exactly one vertex. Therefore, $X(R, S)$ is a block diagonal matrix such that each block of $X(R, S)$ is the incidence matrix of a tree from which one row is omitted. Thus, by Lemma $4, X(R, S)$ is invertible.

Equalities hold in (3) for all $i=0,1, \ldots, n$ if and only if $L$ and $Q$ are similar and this happens if and only if $G$ is a bipartite graph.

Now, our main result follows immediately from Theorem 2 and Theorem 5. 
Theorem 6 Let $G$ be a graph of order $n$. Then for every $\alpha, 0<\alpha \leq 1$,

$$
q_{1}^{\alpha}+\cdots+q_{n}^{\alpha} \geq \mu_{1}^{\alpha}+\cdots+\mu_{n}^{\alpha} .
$$

We conjecture that the equality holds if and only if $G$ is bipartite.

\section{Sum of powers of the Laplacian eigenvalues of graphs}

Let $G$ be a graph and $\mu_{1}, \ldots, \mu_{k}$ be the non-zero Laplacian eigenvalues of $G$. In [10, 11], the sum of powers of the Laplacian eigenvalues of a graph $G$,

$$
S_{\alpha}(G):=\mu_{1}^{\alpha}+\cdots+\mu_{k}^{\alpha},
$$

for $\alpha \neq 0$ was studied. In this section we provide new bounds for $S_{\alpha}(G)$ when $0<$ $\alpha \leq 1$. We denote by $S_{n}$ and $P_{n}$ the star and the path on $n$ vertices, respectively. Let $T$ be a tree of order $n$ and $\sum_{i=0}^{n}(-1)^{i} b_{i}(T) \lambda^{n-i}$ be the characteristic polynomial of its Laplacian. It is known that [12]

$$
b_{i}\left(S_{n}\right) \leq b_{i}(T) \leq b_{i}\left(P_{n}\right), \quad \text { for } i=0, \ldots, n \text {. }
$$

Therefore, we have the following corollary from Theorem 2:

Corollary 7 Let $\alpha, 0<\alpha \leq 1$ be a real number. Then, for any tree of order $n$,

$$
S_{\alpha}\left(S_{n}\right) \leq S_{\alpha}(T) \leq S_{\alpha}\left(P_{n}\right)
$$

The following follows from the Courant-Weyl inequalities (see, e.g., [7, Theorem 4.3.7]).

Lemma 8 Let $G$ be a graph of order $n$ and e be an edge of $G$. Then the Laplacian eigenvalues of $G$ and $G^{\prime}=G-e$ interlace:

$$
\mu_{1}(G) \geq \mu_{1}\left(G^{\prime}\right) \geq \mu_{2}(G) \geq \mu_{2}\left(G^{\prime}\right) \geq \cdots \geq \mu_{n}(G)=\mu_{n}\left(G^{\prime}\right)=0 .
$$

To prove our next result, we recall that the Laplacian eigenvalues of $S_{n}$ are 0 with multiplicity 1,1 with multiplicity $n-2$, and $n$ with multiplicity 1 .

Corollary 9 For any connected graph $G$ of order $n$ and $0<\alpha \leq 1$,

$$
S_{\alpha}(G) \geq n+n^{\alpha}-2 \text {. }
$$

Proof Let $T$ be a spanning tree of $G$, then by Lemma 8 and Corollary 7 ,

$$
S_{\alpha}(G) \geq S_{\alpha}(T) \geq S_{\alpha}\left(S_{n}\right)=n+n^{\alpha}-2 .
$$

The corollary extends a result of [6] in which the special case of the inequality for $\alpha=\frac{1}{2}$ was established. 
Acknowledgements The research of the first author was in part supported by a grant (No. 88050212) from School of Mathematics, Institute for Research in Fundamental Sciences (IPM). This work was done while the second author was visiting the department of mathematics of POSTECH. He would like to thank the department for its hospitality and support. The third author was partially supported by the Priority Research Centers Program through the National Research Foundation of Korea (NRF) funded by the Ministry of Education, Science and Technology (Grant 2009-0094069). The second and fourth authors thank the School of Mathematics, Institute for Research in Fundamental Sciences (IPM) for its support.

\section{References}

1. Akbari, S., Ghorbani, E., Oboudi, M.R.: A conjecture on square roots of Laplacian and signless Laplacian eigenvalues of graphs. arXiv:0905.2118v1 [math.CO]

2. Biggs, N.: Algebraic Graph Theory, 2nd edn. Cambridge Mathematical Library. Cambridge University Press, Cambridge (1993)

3. Efroymson, G.A., Swartz, B., Wendroff, B.: A new inequality for symmetric functions. Adv. Math. 38, 109-127 (1980)

4. Grone, R., Merris, R., Sunder, V.S.: The Laplacian spectrum of a graph. SIAM J. Matrix Anal. Appl. 11, 218-238 (1990)

5. Gutman, I., Kiani, D., Mirzakhah, M.: On incidence energy of graphs. MATCH Commun. Math. Comput. Chem. 62, 573-580 (2009)

6. Gutman, I., Kiani, D., Mirzakhah, M., Zhou, B.: On incidence energy of a graph. Linear Algebra Appl. 431, 1223-1233 (2009)

7. Horn, R.A., Johnson, C.R.: Matrix Analysis. Cambridge University Press, Cambridge (1985)

8. Jooyandeh, M.R., Kiani, D., Mirzakhah, M.: Incidence energy of a graph. MATCH Commun. Math. Comput. Chem. 62, 561-572 (2009)

9. Liu, J., Liu, B.: A Laplacian-energy like invariant of a graph. MATCH Commun. Math. Comput. Chem. 59, 355-372 (2008)

10. Tian, G.-X., Huang, T.-Z., Zhou, B.: A note on sum of powers of the Laplacian eigenvalues of bipartite graphs. Linear Algebra Appl. 430, 2503-2510 (2009)

11. Zhou, B.: On sum of powers of the Laplacian eigenvalues of graphs. Linear Algebra Appl. 429, 22392246 (2008)

12. Zhou, B., Gutman, I.: A connection between ordinary and Laplacian spectra of bipartite graphs. Linear Multilinear Algebra 56, 305-310 (2008) 